\title{
ANTI-HOMOMORPHISMS BETWEEN MODULE LATTICES
}

\author{
PATRICK F. SMITH
}

\begin{abstract}
We examine the properties of certain mappings between the lattice $\mathcal{L}(R)$ of ideals of a commutative ring $R$ and the lattice $\mathcal{L}\left({ }_{R} M\right)$ of submodules of an $R$-module $M$, in particular considering when these mappings are lattice anti-homomorphisms. The mappings in question are the mapping $\alpha: \mathcal{L}(R) \rightarrow \mathcal{L}\left({ }_{R} M\right)$ defined by setting for each ideal $B$ of $R, \alpha(B)$ to be the submodule of $M$ consisting of all elements $m$ in $M$ with $B m=0$ and the mapping $\beta: \mathcal{L}\left({ }_{R} M\right) \rightarrow \mathcal{L}(R)$ defined by $\beta(N)$ is the annihilator in $R$ of $N$, for each submodule $N$ of $M$.
\end{abstract}

1. Introduction. This paper is concerned with mappings, in particular anti-homomorphisms, between certain lattices. Let $L$ and $L^{\prime}$ be lattices. As usual, given $a$ and $b$ in $L$, the least upper bound and the greatest lower bound of $a$ and $b$ are denoted by $a \vee b$ and $a \wedge b$, respectively. Given mappings $\varphi, \theta$ from a lattice $L$ to a lattice $L^{\prime}$ we write $\varphi \leq \theta$ to mean that $\varphi(a) \leq \theta(a)$ for all $a \in L$. Clearly, $\varphi=\theta$ if and only if $\varphi \leq \theta$ and $\theta \leq \varphi$.

A mapping $\varphi$ from a lattice $L$ to a lattice $L^{\prime}$ is an anti-homomorphism, provided

$$
\varphi(a \vee b)=\varphi(a) \wedge \varphi(b) \text { and } \varphi(a \wedge b)=\varphi(a) \vee \varphi(b),
$$

for all $a, b \in L$. In other words, a mapping $\varphi$ from a lattice $L$ to a lattice $L^{\prime}$ is an anti-homomorphism if and only if the mapping $\varphi$ is a homomorphism from $L$ to the opposite lattice of $L^{\prime}$. A bijective (respectively, injective, surjective) anti-homomorphism is called an anti-isomorphism (respectively, anti-monomorphism, anti-epimorphism). The first result is absolutely standard and easy to prove.

2010 AMS Mathematics subject classification. Primary 06A30, 13F05, 13A15, 13C12, $13 \mathrm{C} 99$.

Keywords and phrases. Lattice anti-homomorphism, commutative ring, Dedekind domain, chain ring, comultiplication module, von Neumann regular ring.

Received by the editors on January 24, 2014, and in revised form on May 29, 2014. 
Lemma 1.1. The following statements are equivalent for a bijection $\varphi$ from a lattice $L$ to a lattice $L^{\prime}$.

(i) $\varphi$ is an anti-isomorphism.

(ii) $\varphi(a \vee b)=\varphi(a) \wedge \varphi(b)$ for all $a, b \in L$.

(iii) $\varphi(a \wedge b)=\varphi(a) \vee \varphi(b)$ for all $a, b \in L$.

Moreover, in this case the inverse mapping $\varphi^{-1}: L^{\prime} \rightarrow L$ is also an anti-isomorphism.

Throughout this note, all rings will be commutative with identity, and all modules will be unital. Let $R$ be a ring, and let $M$ be any $R$-module. The collection of submodules of $M$ form a lattice which we shall denote by $\mathcal{L}\left({ }_{R} M\right)$ with respect to the following definitions:

$$
L \vee N=L+N \quad \text { and } \quad L \wedge N=L \cap N,
$$

for all submodules $L$ and $N$ of $M$. Note that $\mathcal{L}\left({ }_{R} M\right)$ is a lattice with least element the zero submodule, greatest element $M$ and, for any given submodules $L$ and $N$ of $M$,

$$
L \leq N \text { in } \mathcal{L}\left({ }_{R} M\right) \Longleftrightarrow L \subseteq N \text { in } M .
$$

In particular, we shall denote the lattice $\mathcal{L}\left({ }_{R} R\right)$ of ideals of $R$ by $\mathcal{L}(R)$. We shall be interested in mappings between $\mathcal{L}(R)$ and $\mathcal{L}\left({ }_{R} M\right)$.

For any ideal $B$ of the $\operatorname{ring} R$ we shall denote by $\operatorname{ann}_{M}(B)$ the set of all elements $m \in M$ such that $B m=0$. Note that $\operatorname{ann}_{M}(B)$ is a submodule of $M$. In addition, for any submodule $N$ of $M$ we denote by $\operatorname{ann}_{R}(N)$ the set of all elements $r \in R$ such that $r N=0$ and note that $\operatorname{ann}_{R}(N)$ is an ideal of $R$. Let $A=\operatorname{ann}_{R}(M)$, the annihilator of $M$ in $R$. By defining

$$
(r+A) m=r m \quad(r \in R, m \in M),
$$

$M$ becomes a faithful $(R / A)$-module with the property that a subset $X$ of $M$ is an $R$-submodule of $M$ if and only if $X$ is an $(R / A)$-submodule of $M$. Thus, the lattice $\mathcal{L}\left({ }_{R} M\right)$ is identical to the lattice $\mathcal{L}\left({ }_{R / A} M\right)$. Note that

$$
\operatorname{ann}_{R / A}(N)=\operatorname{ann}_{R}(N) / A
$$

for every submodule $N$ of $M$. In addition,

$$
\operatorname{ann}_{M}((B+A) / A)=\operatorname{ann}_{M}(B),
$$


for every ideal $B$ of $R$. This implies that

$$
\operatorname{ann}_{M}\left(\operatorname{ann}_{R}(N)\right)=\operatorname{ann}_{M}\left(\operatorname{ann}_{R / A}(N)\right),
$$

for every submodule $N$ of $M$.

Let $L$ and $N$ be any submodules of a module $M$ over a general ring $R$. Then $\left(L:_{R} N\right)$ will denote the set of elements $r \in R$ such that $r N \subseteq L$. Thus, $\left(L:_{R} N\right)=\operatorname{ann}_{R}((N+L) / L)$ which is an ideal of the ring $R$. In $[\mathbf{1 7}, \mathbf{1 8}]$, we investigate the mapping $\lambda: \mathcal{L}(R) \rightarrow \mathcal{L}\left({ }_{R} M\right)$ defined by $\lambda(B)=B M$ for every ideal $B$ of $R$ and the mapping $\mu: \mathcal{L}\left({ }_{R} M\right) \rightarrow \mathcal{L}(R)$ defined by $\mu(N)=\left(N:_{R} M\right)$ for every submodule $N$ of $M$. It is proved that $\lambda=\lambda \mu \lambda$ and $\mu=\mu \lambda \mu$. In $[\mathbf{1 7}, \mathbf{1 8}]$, we examine when the mappings $\lambda$ and $\mu$ are (lattice) homomorphisms with different properties. The module $M$ is called a $\lambda$-module in case the mapping $\lambda$ is a homomorphism and is called a $\mu$-module if $\mu$ is a homomorphism.

Now we define a mapping $\alpha: \mathcal{L}(R) \rightarrow \mathcal{L}\left({ }_{R} M\right)$ by

$$
\alpha(B)=\operatorname{ann}_{M}(B),
$$

for every ideal $B$ of $R$ and we define a mapping $\beta: \mathcal{L}\left({ }_{R} M\right) \rightarrow \mathcal{L}(R)$ by

$$
\beta(N)=\operatorname{ann}_{R}(N),
$$

for every submodule $N$ of $M$. Let $A=\operatorname{ann}_{R}(M)$. We shall denote by $\bar{\alpha}$ the mapping from $\mathcal{L}(R / A)$ to $\mathcal{L}\left({ }_{R} M\right)$ defined by $\bar{\alpha}(B / A)=\operatorname{ann}_{M}(B / A)$ for every ideal $B$ of $R$ containing $A$. Note that, by our above remarks, $\bar{\alpha}(B / A)=\operatorname{ann}_{M}(B)$ for every ideal $B$ containing $A$. In addition, we denote by $\bar{\beta}$ the mapping from $\mathcal{L}\left({ }_{R / A} M\right)$ to $\mathcal{L}(R / A)$ defined by $\bar{\beta}(N)=\operatorname{ann}_{R / A}(N)$ for every submodule $N$ of $M$. Our above remarks show that $\bar{\beta}(N)=\operatorname{ann}_{R}(N) / A$ for every submodule $N$ of $M$. It follows that, if $\pi: R \rightarrow R / A$ is the canonical projection, then $\alpha=\bar{\alpha} \pi$ and $\bar{\beta}=\pi \beta$.

Note the following result.

Lemma 1.2. Let $R$ be a ring, and let $M$ be any $R$-module. Then, with the above notation,

(a) $\alpha(C) \leq \alpha(B)$ for all ideals $B$ and $C$ of $R$ with $B \leq C$ in $\mathcal{L}(R)$.

(b) $\beta(N) \leq \beta(L)$ for all submodules $L$ and $N$ of $M$ with $L \leq N$ in $\mathcal{L}\left({ }_{R} M\right)$. 
(c) $\alpha \beta \alpha=\alpha$.

(d) $\beta \alpha \beta=\beta$.

Proof. (a), (b). Clear.

(c). Let $B$ be any ideal of $R$. Then $B \operatorname{ann}_{M}(B)=0$ so that

$$
B \subseteq \operatorname{ann}_{R}\left(\operatorname{ann}_{M}(B)\right)=\beta \alpha(B) .
$$

It follows that $1 \leq \beta \alpha$. Similarly, $1 \leq \alpha \beta$. By (a), $\alpha \beta \alpha(B)=$ $\alpha(\beta \alpha(B)) \leq \alpha(B)$. Thus, $\alpha \beta \alpha \leq \alpha$. On the other hand, $1 \leq \alpha \beta$ implies that $\alpha(B) \leq \alpha \beta(\alpha(B))=\alpha \beta \alpha(B)$. This gives that $\alpha \leq \alpha \beta \alpha$, and hence, $\alpha=\alpha \beta \alpha$.

(d). Similar to (c).

Lemma 1.2 has as an easy consequence the following result.

Lemma 1.3. With the above notation, the following statements are equivalent:

(i) $\alpha$ is a surjection.

(ii) $\alpha \beta=1$.

(iii) $N=\operatorname{ann}_{M}\left(\operatorname{ann}_{R}(N)\right)$ for every submodule $N$ of $M$.

(iv) $\beta$ is an injection.

(v) $\bar{\alpha}$ is a surjection.

(vi) $\bar{\alpha} \bar{\beta}=1$.

(vii) $\bar{\beta}$ is an injection.

Proof. (i) $\Rightarrow$ (ii). By Lemma 1.2(c).

(ii) $\Rightarrow$ (i), (iv). Clear.

(ii) $\Leftrightarrow$ (iii). Clear.

(iv) $\Rightarrow$ (ii). By Lemma 1.2(d).

(iii) $\Leftrightarrow$ (v) $\Leftrightarrow$ (vi) $\Leftrightarrow$ (vii). We have noted above that

$$
\operatorname{ann}_{M}\left(\operatorname{ann}_{R}(N)\right)=\operatorname{ann}_{M}\left(\operatorname{ann}_{R / A}(N)\right),
$$

for every submodule $N$ of $M$ and thus (iii) $\Leftrightarrow$ (v) $\Leftrightarrow$ (vi) $\Leftrightarrow$ (vii) all follow from the equivalence of (i)-(iv). 
The proof of the next result is similar to the proof of Lemma 1.3. For the last part, note that if $M$ is an $R$-module with $A=\operatorname{ann}_{R}(M)$, then

$$
\alpha(A)=\operatorname{ann}_{M}(A)=M=\alpha(0) .
$$

Lemma 1.4. With the above notation, the following statements are equivalent:

(i) $\alpha$ is an injection.

(ii) $\beta \alpha=1$.

(iii) $B=\operatorname{ann}_{R}\left(\operatorname{ann}_{M}(B)\right)$ for every ideal $B$ of $R$.

(iv) $\beta$ is a surjection.

Moreover, in this case $M$ is faithful.

Proposition 1.5. With the above notation, the mapping $\alpha$ is a bijection if and only if $\beta$ is a bijection. In this case $\alpha$ and $\beta$ are inverses of each other and are both anti-isomorphisms.

Proof. By Lemmas 1.3 and $1.4 \alpha$ is a bijection if and only if $\beta$ is a bijection and, in this case, $\alpha$ and $\beta$ are inverses of each other. Moreover, for all ideals $B$ and $C$ of $R$,

$$
\begin{aligned}
\alpha(B \vee C) & =\operatorname{ann}_{M}(B+C)=\operatorname{ann}_{M}(B) \cap \operatorname{ann}_{M}(C) \\
& =\alpha(B) \wedge \alpha(C) .
\end{aligned}
$$

By Lemma 1.1, $\alpha$ is an anti-isomorphism. The same proof proves that $\beta$ is also an anti-isomorphism.

Next we recall the definition of trivial extensions. These will provide a fruitful source of examples later. Let $R$ be a ring, and let $M$ be an $R$-module. Then the trivial extension of $M$ by $R$ is the $\operatorname{ring} M \ltimes R$ defined as follows. The set $M \ltimes R$ consists of all ordered pairs $(r, m)$, with $r \in R$ and $m \in M$, and addition and multiplication are defined by

$$
\left(r_{1}, m_{1}\right)+\left(r_{2}, m_{2}\right)=\left(r_{1}+r_{2}, m_{1}+m_{2}\right),
$$

and

$$
\left(r_{1}, m_{1}\right)\left(r_{2}, m_{2}\right)=\left(r_{1} r_{2}, r_{1} m_{2}+r_{2} m_{1}\right),
$$

for all $r_{1}, r_{2} \in R$ and $m_{1}, m_{2} \in M$. It is well known that $M \ltimes R$ is a commutative ring with zero $(0,0)$ and identity $(1,0)$. For any ideal $B$ 
of $R$ and submodule $N$ of the $R$-module $M, N \ltimes B$ will denote the set of elements $(b, x)$ with $b \in B$ and $x \in N$. Note that $N \ltimes B$ is an ideal of $M \ltimes R$ if and only if $B M \subseteq N$.

Let $R$ be any ring, and let $M$ be an $R$-module. We shall call $M$ an $\alpha$-module in case the above mapping $\alpha: \mathcal{L}(R) \rightarrow \mathcal{L}\left({ }_{R} M\right)$ is an antihomomorphism. The $R$-module $M$ is called a comultiplication module in the case where $\alpha$ is a surjection. Thus, $M$ is a comultiplication $\alpha$ module precisely when $\alpha$ is an anti-epimorphism. Note that $M$ is a comultiplication module provided that, for each submodule $N$ of $M$, there exists an ideal $B$ of $R$ such that $N=\operatorname{ann}_{M}(B)$. Comultiplication modules have been studied by a number of authors (see, for example, $[\mathbf{3}, \mathbf{4}, \mathbf{5}, \mathbf{8}, \mathbf{1 0}, \mathbf{1 4}, \mathbf{1 5}])$. In Theorem 4.3 , we characterize when a direct sum of modules is a comultiplication module.

We prove that if $R$ is any ring, then every semisimple $R$-module is an $\alpha$-module (Corollary 2.9). In the case where $R$ is a Dedekind domain or a chain ring or the trivial extension $X \ltimes S$ of the injective envelope $X$ of a simple $S$-module by a Dedekind domain $S$, then every $R$-module is an $\alpha$-module (Corollaries 2.14 and 2.15 and Example 3.15). A ring $R$ is von Neumann regular if and only if the mapping $\alpha$ is an anti-monomorphism where $M$ is the direct sum $\oplus(R / P)$ where $P$ runs through the collection of all prime ideals of $R$ (Theorem 4.7).

2. The mapping $\alpha$. Let $R$ be a ring, and let $M$ be an $R$-module. Recall that we call $M$ an $\alpha$-module in case the mapping $\alpha: \mathcal{L}(R) \rightarrow$ $\mathcal{L}\left({ }_{R} M\right)$ defined by $\alpha(B)=\operatorname{ann}_{M}(B)$ is a (lattice) anti-homomorphism. Note the following result.

Lemma 2.1. The following statements are equivalent for an $R$-module $M$.

(i) $M$ is an $\alpha$-module.

(ii) $\operatorname{ann}_{M}(B \cap C) \subseteq \operatorname{ann}_{M}(B)+\operatorname{ann}_{M}(C)$ for all ideals $B, C$ of $R$.

(iii) $\operatorname{ann}_{M}\left(B_{1} \cap \cdots \cap B_{n}\right)=\operatorname{ann}_{M}\left(B_{1}\right)+\cdots+\operatorname{ann}_{M}\left(B_{n}\right)$ for every positive integer $n$ and ideals $B_{i}(1 \leq i \leq n)$ of $R$.

Proof. (i) $\Rightarrow$ (ii). Let $B$ and $C$ be any ideals of $R$. Then

$$
\begin{aligned}
\operatorname{ann}_{M}(B \cap C) & =\alpha(B \wedge C)=\alpha(B) \vee \alpha(C) \\
& =\operatorname{ann}_{M}(B)+\operatorname{ann}_{M}(C) .
\end{aligned}
$$


(ii) $\Rightarrow$ (iii). Let $n$ be a positive integer, and let $B_{i}(1 \leq i \leq n)$ be any collection of ideals of $R$. Clearly, $\operatorname{ann}_{M}\left(B_{i}\right) \subseteq \operatorname{ann}_{M}\left(B_{1} \cap \cdots \cap B_{n}\right)$ for each $1 \leq i \leq n$. Hence,

$$
\operatorname{ann}_{M}\left(B_{1}\right)+\cdots+\operatorname{ann}_{M}\left(B_{n}\right) \subseteq \operatorname{ann}_{M}\left(B_{1} \cap \cdots \cap B_{n}\right) .
$$

However, by (ii) and induction,

$$
\operatorname{ann}_{M}\left(B_{1} \cap \cdots \cap B_{n}\right) \subseteq \operatorname{ann}_{M}\left(B_{1}\right)+\cdots+\operatorname{ann}_{M}\left(B_{n}\right) .
$$

This proves (iii).

(iii) $\Rightarrow$ (i). Let $B$ and $C$ be any ideals of $R$. Then

$$
\alpha(B \vee C)=\operatorname{ann}_{M}(B+C)=\operatorname{ann}_{M}(B) \cap \operatorname{ann}_{M}(C)=\alpha(B) \wedge \alpha(C) .
$$

Moreover, (iii) gives that

$$
\alpha(B \wedge C)=\operatorname{ann}_{M}(B \cap C)=\operatorname{ann}_{M}(B)+\operatorname{ann}_{M}(C)=\alpha(B) \vee \alpha(C) .
$$

Thus, $\alpha$ is a homomorphism and $M$ is an $\alpha$-module.

Lemma 2.1 has many consequences, and we give a few of these next.

Corollary 2.2. Let $A$ be an ideal of a ring $R$. Then the cyclic $R$ module $R / A$ is an $\alpha$-module if and only if $\left(A:_{R} B \cap C\right)=\left(A:_{R}\right.$ $B)+\left(A:_{R} C\right)$ for all ideals $B$ and $C$ of $R$.

Proof. Let $G$ be any ideal of $R$. Then

$$
\begin{aligned}
\operatorname{ann}_{R / A}(G) & =\{r+A: G(r+A)=0\} \\
& =\{r+A: G r \subseteq A\}=\left(A:_{R} G\right) / A .
\end{aligned}
$$

The result follows by Lemma 2.1 .

Corollary 2.3. Let $R$ be a ring, and let an $R$-module $M$ be an $\alpha$ module with $A=\operatorname{ann}_{R}(M)$. Then $M=\operatorname{ann}_{M}(B)+\operatorname{ann}_{M}(C)$ for all ideals $B$ and $C$ of $R$ with $B \cap C \subseteq A$.

Proof. By Lemma 2.1,

$$
M=\operatorname{ann}_{M}(B \cap C)=\operatorname{ann}_{M}(B)+\operatorname{ann}_{M}(C) .
$$

Recall that a module $M$ is called hollow in the case where $M \neq L+N$ for any proper submodules $L, N$. 
Corollary 2.4. Let $R$ be a ring, and let an $R$-module $M$ be a hollow $\alpha$-module with $A=\operatorname{ann}_{R}(M)$. Let $B$ and $C$ be ideals of $R$ such that $B \cap C \subseteq A$. Then $B \subseteq A$ or $C \subseteq A$.

Proof. By Corollary 2.3, either $M=\operatorname{ann}_{M}(B)$ or $M=\operatorname{ann}_{M}(C)$. Thus, $B M=0$ and $B \subseteq A$ or $C M=0$ and $C \subseteq A$.

Corollary 2.5. Let $M$ be a module over a ring $R$ such that $R m$ is an $\alpha$-module for each element $m \in M$. Then $M$ is an $\alpha$-module.

Proof. Let $B$ and $C$ be ideals of $R$. Let $m \in \operatorname{ann}_{M}(B \cap C)$. By hypothesis and Lemma 2.1,

$$
\begin{aligned}
m \in \operatorname{ann}_{R m}(B \cap C) & =\operatorname{ann}_{R m}(B)+\operatorname{ann}_{R m}(C) \\
& \subseteq \operatorname{ann}_{M}(B)+\operatorname{ann}_{M}(C) .
\end{aligned}
$$

Thus, $\operatorname{ann}_{M}(B \cap C) \subseteq \operatorname{ann}_{M}(B)+\operatorname{ann}_{M}(C)$. By Lemma 2.1, $M$ is an $\alpha$-module.

We next consider some examples of $\alpha$-modules.

Proposition 2.6. Let $R$ be a ring, and let an $R$-module $M=\oplus_{i \in I} M_{i}$ be a direct sum of submodules $M_{i}(i \in I)$. Then $M$ is an $\alpha$-module if and only if $M_{i}$ is an $\alpha$-module for all $i \in I$.

Proof. Suppose first that $M_{i}$ is an $\alpha$-module for all $i \in I$. Let $B$ and $C$ be any ideals of $R$. Then

$$
\begin{aligned}
\operatorname{ann}_{M}(B \cap C) & =\oplus_{i \in I}\left[\operatorname{ann}_{M_{i}}(B \cap C)\right] \\
& =\oplus_{i \in I}\left[\operatorname{ann}_{M_{i}}(B)+\operatorname{ann}_{M_{i}}(C)\right] \\
& =\left[\oplus_{i \in I} \operatorname{ann}_{M_{i}}(B)\right]+\left[\oplus_{i \in I} \operatorname{ann}_{M_{i}}(C)\right] \\
& =\operatorname{ann}_{M}(B)+\operatorname{ann}_{M}(C) .
\end{aligned}
$$

By Lemma 2.1, $M$ is an $\alpha$-module. 
Conversely, suppose that $M$ is an $\alpha$-module. Let $i \in I$, and let $K=M_{i}$. Given any ideals $B$ and $C$ of $R$ we have:

$$
\begin{aligned}
\operatorname{ann}_{K}(B \cap C) & =K \cap\left[\operatorname{ann}_{M}(B \cap C)\right]=K \cap\left[\operatorname{ann}_{M}(B)+\operatorname{ann}_{M}(C)\right] \\
& =\left[K \cap \operatorname{ann}_{M}(B)\right]+\left[K \cap \operatorname{ann}_{M}(C)\right] \\
& =\operatorname{ann}_{K}(B)+\operatorname{ann}_{K}(C) .
\end{aligned}
$$

Thus, $\operatorname{ann}_{K}(B \cap C)=\operatorname{ann}_{K}(B)+\operatorname{ann}_{K}(C)$ for all ideals $B$ and $C$ of $R$. By Lemma 2.1, $K$ is an $\alpha$-module. It follows that $M_{i}$ is an $\alpha$-module for every $i \in I$.

Let $R$ be any ring. An $R$-module $M$ is called prime in the case when $M$ is non-zero and whenever $r m=0$ for some $r \in R, 0 \neq m \in M$ then $r M=0$. It is well known that the module $M$ is prime if and only if $A=\operatorname{ann}_{R}(M)$ is a prime ideal of $R$ and the $(R / A)$-module $M$ is torsionfree. In particular, if $P$ is any prime ideal of $R$, then the $R$-module $R / P$ is prime. There is an extensive literature on the topic of prime modules over commutative rings (see, for example, $[\mathbf{6}, \mathbf{9}, \mathbf{1 1}, \mathbf{1 2}, \mathbf{1 3}]$ ).

Proposition 2.7. Let $R$ be any ring. Then every prime $R$-module is an $\alpha$-module.

Proof. Let $M$ be a prime $R$-module. Let $B$ and $C$ be ideals of $R$. Let $m \in \operatorname{ann}_{M}(B \cap C)$. Then $(B \cap C) m=0$, and hence, $B(C m)=0$. Either $C m=0$ or $B M=0$, in which case $B m=0$. In any case, $m \in \operatorname{ann}_{R}(B) \cup \operatorname{ann}_{R}(C) \subseteq \operatorname{ann}_{R}(B)+\operatorname{ann}_{R}(C)$. By Lemma 2.1, $M$ is an $\alpha$-module.

Corollary 2.8. Let $R$ be any ring. Then every direct sum of prime $R$-modules is an $\alpha$-module.

Proof. By Propositions 2.6 and 2.7.

Corollary 2.9. Let $R$ be any ring. Then every semisimple $R$-module is an $\alpha$-module.

Proof. By Corollary 2.8 because every simple $R$-module is prime.

Next we consider the case of domains. 
Lemma 2.10. Let $R$ be a domain, and let $M$ be an $R$-module with torsion submodule $T$. Then $M$ is an $\alpha$-module if and only if $T$ is an $\alpha$-module.

Proof. Let $G$ be any non-zero ideal of $R$. Clearly, $\operatorname{ann}_{M}(G)=$ $\operatorname{ann}_{T}(G)$. Apply Lemma 2.1.

Corollary 2.11. Let $R$ be a domain, and let $M$ be an $R$-module whose torsion submodule is semisimple. Then $M$ is an $\alpha$-module.

Proof. By Lemma 2.10 and Corollary 2.9 .

Next we investigate rings $R$ with the property that every $R$-module is an $\alpha$-module.

Theorem 2.12. The following statements are equivalent for ideals $B$ and $C$ of a ring $R$.

(i) $\operatorname{ann}_{M}(B \cap C)=\operatorname{ann}_{M}(B)+\operatorname{ann}_{M}(C)$ for every $R$-module $M$.

(ii) $\operatorname{ann}_{M}(B \cap C)=\operatorname{ann}_{M}(B)+\operatorname{ann}_{M}(C)$ for every cyclic $R$-module $M$.

(iii) $R=\left(B:_{R} C\right)+\left(C:_{R} B\right)$.

Proof. (i) $\Rightarrow$ (ii). Clear.

(ii) $\Rightarrow$ (iii). Let $M$ denote the cyclic $R$-module $R /(B \cap C)$. By hypothesis,

$$
R /(B \cap C)=\operatorname{ann}_{M}(B \cap C)=\operatorname{ann}_{M}(B)+\operatorname{ann}_{M}(C) .
$$

But it is easy to check that

$$
\operatorname{ann}_{M}(B)=\{r+(B \cap C): B r \subseteq(B \cap C)\}=\left(C:_{R} B\right) /(B \cap C) .
$$

Similarly, $\operatorname{ann}_{M}(C)=\left(B:_{R} C\right) /(B \cap C)$. It follows that $R=\left(B:_{R}\right.$ $C)+\left(C:_{R} B\right)$.

(iii) $\Rightarrow$ (i). There exist elements $u \in\left(B:_{R} C\right)$ and $v \in\left(C:_{R} B\right)$ such that $1=u+v$. Let $M$ be any $R$-module, and let $m \in \operatorname{ann}_{M}(B \cap C)$. Note that $B(v m)=(v B) m \subseteq(B \cap C) m=0$ so that $v m \in \operatorname{ann}_{M}(B)$. Similarly, $u m \in \operatorname{ann}_{M}(C)$. Thus, $m=v m+u m \in \operatorname{ann}_{M}(B)+$ $\operatorname{ann}_{M}(C)$. We have proved that $\operatorname{ann}_{M}(B \cap C) \subseteq \operatorname{ann}_{M}(B)+\operatorname{ann}_{M}(C)$. Hence, $\operatorname{ann}_{M}(B \cap C)=\operatorname{ann}_{M}(B)+\operatorname{ann}_{M}(C)$. 
Corollary 2.13. Let $R$ be a ring. Then the following statements are equivalent:

(i) Every $R$-module is an $\alpha$-module.

(ii) Every cyclic $R$-module is an $\alpha$-module.

(iii) $R=\left(B:_{R} C\right)+\left(C:_{R} B\right)$ for all ideals $B$ and $C$ of $R$.

Proof. By Lemma 2.1 and Theorem 2.12 .

Corollary 2.14. A Noetherian domain $R$ is Dedekind if and only if every $R$-module is an $\alpha$-module.

Proof. By Corollary 2.13 and [7, Theorem 25.2].

Given a ring $R$, we shall call an $R$-module $M$ a chain module in case the submodules of $M$ are linearly ordered. That is, $M$ is a chain module if and only if $L \subseteq N$ or $N \subseteq L$ for all submodules $L, N$ of $M$. The ring $R$ is called a chain ring in the case where the $R$-module $R$ is a chain module. A ring $R$ is called local if $R$ contains a unique maximal ideal. Clearly, chain rings are local.

Corollary 2.15. A ring $R$ is a chain ring if and only if $R$ is local and every $R$-module is an $\alpha$-module.

Proof. Suppose first that $R$ is a chain ring. Then $R$ is a local ring. Let $B$ and $C$ be any ideals of $R$. Then $B \subseteq C$, and hence $\left(C:_{R} B\right)=R$, or else $C \subseteq B$ and in this case $\left(B:_{R} C\right)=R$. In any case, $R=\left(B:_{R} C\right)+\left(C:_{R} B\right)$. By Corollary 2.13 every $R$-module is an $\alpha$-module. Conversely, suppose that $R$ is a local ring such that every $R$-module is an $\alpha$-module. Let $G$ and $H$ be ideals of $R$. By Corollary 2.13, $R=\left(G:_{R} H\right)+\left(H:_{R} G\right)$. But $R$ is a local ring so that $R=\left(G:_{R} H\right)$, and hence $H \subseteq G$, or else $R=\left(H:_{R} G\right)$ and in this case $G \subseteq H$. This proves that $R$ is a chain ring.

A ring $R$ need not be a chain ring nor a Dedekind domain in order that every $R$-module be an $\alpha$-module. For example, we have the following result.

Proposition 2.16. Let a ring $R=R_{1} \oplus \cdots \oplus R_{n}$ be a direct sum of subrings $R_{i}(1 \leq i \leq n)$, for some positive integer $n$. Suppose that, for 
each $1 \leq i \leq n$, every $R_{i}$-module is an $\alpha$-module. Then every $R$-module is an $\alpha$-module.

Proof. Let $B$ and $C$ be any ideals of $R$. Then $B=B_{1} \oplus \cdots \oplus B_{n}$ for some ideal $B_{i}$ of $R_{i}$ for all $1 \leq i \leq n$. Similarly, $C=C_{1} \oplus \cdots \oplus C_{n}$ for some ideal $C_{i}$ of $R_{i}$ for each $1 \leq i \leq n$. This implies that

$$
\left(B:_{R} C\right)=\left(B_{1}:_{R_{1}} C_{1}\right) \oplus \cdots \oplus\left(B_{n}:_{R_{n}} C_{n}\right),
$$

and

$$
\left(C:_{R} B\right)=\left(C_{1}:_{R_{1}} B_{1}\right) \oplus \cdots \oplus\left(C_{n}:_{R_{n}} B_{n}\right) .
$$

By Corollary 2.13, $R_{i}=\left(B_{i}:_{R_{i}} C_{i}\right)+\left(C_{i}:_{R_{i}} B_{i}\right)$ for all $1 \leq i \leq n$. Thus, $\left(B:_{R} C\right)+\left(C:_{R} B\right)=R_{1} \oplus \cdots \oplus R_{n}=R$. We have proved that $R=\left(B:_{R} C\right)+\left(C:_{R} B\right)$ for all ideals $B, C$ of $R$, and hence, by Corollary 2.13 , every $R$-module is an $\alpha$-module.

3. The mapping $\beta$. Let $R$ be a ring, and let $M$ be an $R$-module. Recall that $\beta$ is the mapping from $\mathcal{L}\left({ }_{R} M\right)$ to $\mathcal{L}(R)$ defined by $\beta(N)=$ $\operatorname{ann}_{R}(N)$ for every submodule $N$ of $M$. The module $M$ is called a $\beta$-module in case $\beta$ is an anti-homomorphism. The proof of the next result is very similar to that of Lemma 2.1 and so is omitted.

Lemma 3.1. Let $R$ be any ring. Then the following statements are equivalent for an $R$-module $M$.

(i) $M$ is a $\beta$-module.

(ii) $\operatorname{ann}_{R}(L \cap N) \subseteq \operatorname{ann}_{R}(L)+\operatorname{ann}_{R}(N)$ for all submodules $L$ and $N$ of $M$.

(iii) $\operatorname{ann}_{R}\left(L_{1} \cap \cdots \cap L_{n}\right)=\operatorname{ann}_{R}\left(L_{1}\right)+\cdots+\operatorname{ann}_{R}\left(L_{n}\right)$ for every positive integer $n$ and submodules $L_{i}(1 \leq i \leq n)$ of $M$.

Corollary 3.2. For any ring $R$, every submodule of a $\beta$-module over $R$ is also a $\beta$-module.

Proof. By Lemma 3.1.

Corollary 3.3. Let $R$ be any ring. Then the $R$-module $R$ is an $\alpha$ module if and only if it is a $\beta$-module.

Proof. By Lemmas 2.1 and 3.1. 
In contrast to Corollary 2.9, we note the following fact.

Corollary 3.4. Let $R$ be a ring, and let $M$ be any $R$-module such that there exists a non-zero $R$-module $X$ with the property that the $R$-module $X \oplus X$ embeds in $M$. Then the $R$-module $M$ is not a $\beta$-module.

Proof. There exist submodules $L$ and $N$ of $M$ such that $L \cap N=0$ and $L \cong N \cong X$. Thus, $\operatorname{ann}_{R}(L \cap N)=R \neq \operatorname{ann}_{R}(X)=\operatorname{ann}_{R}(L)+$ $\operatorname{ann}_{R}(N)$. Apply Lemma 3.1.

We next look at homomorphic images and have the following result.

Theorem 3.5. The following statements are equivalent for a module $M$ over a general ring $R$.

(i) Every homomorphic image of $M$ is a $\beta$-module.

(ii) Every submodule of $M$ is a $\mu$-module.

(iii) $R=\left(L:_{R} N\right)+\left(N:_{R} L\right)$ for all submodules $L$ and $N$ of $M$.

Proof. (i) $\Rightarrow$ (iii). Let $L$ and $N$ be any submodules of $M$. Then $M /(L \cap N)$ is a $\beta$-module. By Lemma 3.1,

$$
\begin{aligned}
R & =\operatorname{ann}_{R}((L /(L \cap N)) \cap(N /(L \cap N))) \\
& =\operatorname{ann}_{R}(L /(L \cap N))+\operatorname{ann}_{R}(N /(L \cap N)) \\
& =\left(N:_{R} L\right)+\left(L:_{R} N\right) .
\end{aligned}
$$

(iii) $\Rightarrow$ (i). Let $K$ be any proper submodule of $M$. Let $\bar{M}$ denote the $R$-module $M / K$. Let $\bar{L}$ and $\bar{N}$ be any submodules of $\bar{M}$. Then there exist submodules $L$ and $N$ of $M$, each containing $K$, such that $\bar{L}=L / K$ and $\bar{N}=N / K$. Note that

$$
\operatorname{ann}_{R}(\bar{L} \cap \bar{N})=\operatorname{ann}_{R}((L \cap N) / K)=\left(K:_{R} L \cap N\right) .
$$

By hypothesis, $R=\left(N:_{R} L\right)+\left(L:_{R} N\right)$, and hence

$$
\begin{aligned}
\left(K:_{R} L \cap N\right)= & \left(K:_{R} L \cap N\right)\left(N:_{R} L\right) \\
& +\left(K:_{R} L \cap N\right)\left(L:_{R} N\right) \\
\subseteq & \left(K:_{R} L\right)+\left(K:_{R} N\right) \\
= & \operatorname{ann}_{R}(\bar{L})+\operatorname{ann}_{R}(\bar{N}) .
\end{aligned}
$$


By Lemma 3.1 , the $R$-module $\bar{M}$ is a $\beta$-module.

(ii) $\Leftrightarrow$ (iii). By [17, Lemma 3.14].

Corollary 3.6. Let $R$ be any ring. If an $R$-module $M$ is a chain module, then every homomorphic image of $M$ is a $\beta$-module. Moreover, the converse holds if $R$ is a local ring.

Proof. Suppose that $M$ is a chain module. Let $L$ and $N$ be submodules of $M$. Then $L \subseteq N$ or $N \subseteq L$. Without loss of generality, $L \subseteq N$. Then $L \cap N=L$, and we have the following:

$$
\operatorname{ann}_{R}(L \cap N)=\operatorname{ann}_{R}(L)=\operatorname{ann}_{R}(L)+\operatorname{ann}_{R}(N),
$$

because $\operatorname{ann}_{R}(N) \subseteq \operatorname{ann}_{R}(L)$. By Proposition 3.5, every homomorphic image of $M$ is a $\beta$-module.

Conversely, suppose that $R$ is a local ring and every homomorphic image of $M$ is a $\beta$-module. Let $L$ and $N$ be any submodules of $M$. By Proposition 3.5, $R=\left(L:_{R} N\right)+\left(N:_{R} L\right)$. Because $R$ is a local ring, either $R=\left(L:_{R} N\right)$ and $N \subseteq L$ or $R=\left(N:_{R} L\right)$ and $L \subseteq N$. It follows that $M$ is a chain module.

We now look at direct sums of modules. Note the following result.

Lemma 3.7. Let $B_{1}, B_{2}, C_{1}$ and $C_{2}$ be ideals of a ring $R$ such that

$$
R=B_{1}+C_{2}=B_{2}+C_{1} .
$$

Then

$$
\left(B_{1}+C_{1}\right) \cap\left(B_{2}+C_{2}\right)=\left(B_{1} \cap B_{2}\right)+\left(C_{1} \cap C_{2}\right) .
$$

Proof. It is clear that $\left(B_{1} \cap B_{2}\right)+\left(C_{1} \cap C_{2}\right) \subseteq\left(B_{1}+C_{1}\right) \cap\left(B_{2}+C_{2}\right)$. Next, note that $B_{1}+C_{1}=\left(B_{1}+C_{1}\right)\left(B_{2}+C_{1}\right) \subseteq B_{1} B_{2}+C_{1}$, and hence, $B_{1}+C_{1}=B_{1} B_{2}+C_{1}$. Similarly, $B_{2}+C_{2}=B_{2}+C_{1} C_{2}$. Therefore, we have by the modular law,

$$
\begin{aligned}
\left(B_{1}+C_{1}\right) \cap\left(B_{2}+C_{2}\right) & =\left(B_{1} B_{2}+C_{1}\right) \cap\left(B_{2}+C_{1} C_{2}\right) \\
& =\left[\left(B_{1} B_{2}+C_{1}\right) \cap B_{2}\right]+C_{1} C_{2} \\
& =B_{1} B_{2}+\left(B_{2} \cap C_{1}\right)+C_{1} C_{2} .
\end{aligned}
$$


However, $R=B_{1}+C_{2}=B_{2}+C_{1}$ implies that

$$
B_{2} \cap C_{1}=B_{2} C_{1}=B_{2} C_{1}\left(B_{1}+C_{2}\right) \subseteq B_{1} B_{2}+C_{1} C_{2} .
$$

It follows that $\left(B_{1}+C_{1}\right) \cap\left(B_{2}+C_{2}\right) \subseteq B_{1} B_{2}+C_{1} C_{2} \subseteq\left(B_{1} \cap B_{2}\right)+$ $\left(C_{1} \cap C_{2}\right)$.

Theorem 3.8. Let $R$ be any ring, and let an $R$-module $M=M_{1} \oplus$ $\cdots \oplus M_{k}$ be the direct sum of submodules $M_{i}(1 \leq i \leq k)$ for some positive integer $k$. Then $M$ is a $\beta$-module if and only if $M_{i}$ is a $\beta$ module for each $1 \leq i \leq k$ and $R=\operatorname{ann}_{R}\left(M_{i}\right)+\operatorname{ann}_{R}\left(M_{j}\right)$ for all integers $1 \leq i<j \leq k$.

Proof. Suppose first that $M$ is a $\beta$-module. By Corollary 3.2, the submodule $M_{i}$ is a $\beta$-module for all $1 \leq i \leq k$. Moreover, for all $1 \leq i<j \leq k$, Lemma 3.1 gives that $R=\operatorname{ann}_{R}\left(M_{i} \cap M_{j}\right)=$ $\operatorname{ann}_{R}\left(M_{i}\right)+\operatorname{ann}_{R}\left(M_{j}\right)$.

Conversely, suppose the submodules $M_{i}(1 \leq i \leq k)$ satisfy the stated conditions. By induction on $k$, it is sufficient to prove the result when $k=2$. Let $L$ and $N$ be any submodules of $M$. Let $x \in L$. Then $x=x_{1}+x_{2}$ for some $x_{1} \in M_{1}$ and $x_{2} \in M_{2}$. There exist elements $a_{1}$ and $a_{2}$ in $R$ such that $1=a_{1}+a_{2}, a_{1} M_{1}=0$ and $a_{2} M_{2}=0$. It follows that $x_{1}=\left(1-a_{1}\right) x_{1}=a_{2} x_{1}=a_{2} x \in L \cap M_{1}$. Similarly, $x_{2} \in L \cap M_{2}$, so that $x \in\left(L \cap M_{1}\right)+\left(L \cap M_{2}\right)$. If $L_{1}=L \cap M_{1}$ and $L_{2}=L \cap M_{2}$, then $L_{1}$ is a submodule of $M_{1}, L_{2}$ is a submodule of $M_{2}$ and $L=L_{1} \oplus L_{2}$. Similarly, $N=N_{1} \oplus N_{2}$ for some submodules $N_{1}$ of $M_{1}$ and $N_{2}$ of $M_{2}$. Let $B_{i}=\operatorname{ann}_{R}\left(L_{i}\right)(i=1,2)$, and let $C_{i}=\operatorname{ann}_{R}\left(N_{i}\right)(i=1,2)$. Note that

$$
R=\operatorname{ann}_{R}\left(M_{1}\right)+\operatorname{ann}_{R}\left(M_{2}\right) \subseteq \operatorname{ann}_{R}\left(L_{1}\right)+\operatorname{ann}_{R}\left(N_{2}\right)=B_{1}+C_{2} .
$$

Thus, $R=B_{1}+C_{2}$. Similarly, $R=B_{2}+C_{1}$. Next, $L \cap N=$ $\left(L_{1} \cap N_{1}\right) \oplus\left(L_{2} \cap N_{2}\right)$, and hence, we have by hypothesis and Lemma 3.1,

$$
\begin{aligned}
\operatorname{ann}_{R}(L \cap N) & =\operatorname{ann}_{R}\left(L_{1} \cap N_{1}\right) \cap \operatorname{ann}_{R}\left(L_{2} \cap N_{2}\right) \\
& =\left[\operatorname{ann}_{R}\left(L_{1}\right)+\operatorname{ann}_{R}\left(N_{1}\right)\right] \cap\left[\operatorname{ann}_{R}\left(L_{2}\right)+\operatorname{ann}_{R}\left(N_{2}\right)\right] \\
& =\left(B_{1}+C_{1}\right) \cap\left(B_{2}+C_{2}\right) .
\end{aligned}
$$

But Lemma 3.7 now gives

$$
\left(B_{1}+C_{1}\right) \cap\left(B_{2}+C_{2}\right) \subseteq\left(B_{1} \cap B_{2}\right)+\left(C_{1} \cap C_{2}\right)=\operatorname{ann}_{R}(L)+\operatorname{ann}_{R}(N) .
$$


We have proved that $\operatorname{ann}_{R}(L \cap N) \subseteq \operatorname{ann}_{R}(L)+\operatorname{ann}_{R}(N)$ for all submodules $L$ and $N$ of $M$. By Lemma 3.1, the $R$-module $M$ is a $\beta$-module.

Recall that, for any ring $R$, an $R$-module $U$ is called uniform in the case where $U$ is non-zero and $X \cap Y \neq 0$ for all non-zero submodules $X$ and $Y$ of $U$. Recall further that a submodule $L$ of an $R$-module $M$ is essential provided $L \cap N \neq 0$ for every non-zero submodule $N$ of $M$. We shall apply Theorem 3.8 to modules over Dedekind domains. First we note a simple fact.

Lemma 3.9. Let $R$ be a ring, and let $M$ be an $R$-module such that $M$ is a $\beta$-module. Then every submodule $L$ of $M$ with $\operatorname{ann}_{R}(L)=0$ is essential in $M$.

Proof. Let $N$ be any submodule of $M$ with $L \cap N=0$. By Lemma 3.1, $R=\operatorname{ann}_{R}(L \cap N)=\operatorname{ann}_{R}(L)+\operatorname{ann}_{R}(N)=\operatorname{ann}_{R}(N)$. It follows that $N=0$. Hence, $L$ is an essential submodule of $M$.

Proposition 3.10. Let $R$ be any domain, and let $M$ be a non-zero $R$-module. Then $M$ is a $\beta$-module which is not a torsion $R$-module if and only if $M$ is a torsion-free uniform module.

Proof. Suppose that $M$ is a $\beta$-module which is not a torsion module. Let $m$ be any element of $M$ which is not a torsion element. Then $R m \cong R$ so that $R m$ is a uniform module. Moreover, $\operatorname{ann}_{R}(R m)=0$ implies that $R m$ is essential in $M$ by Lemma 3.9. Thus, $M$ is a uniform module and is torsion-free. Conversely, suppose that $M$ is a torsion-free uniform module. Then $M$ is definitely not a torsion module. Clearly, $\beta(K)=0$ for every non-zero submodule $K$ of $M$, and it easily follows that $\beta$ is a homomorphism because $M$ is uniform. Thus, $M$ is a $\beta$ module.

The next result characterizes $\beta$-modules over a Dedekind domain. Recall that Corollary 2.14 shows that every module over a Dedekind domain is an $\alpha$-module. If $R$ is any ring, $B$ an ideal of $R$ and $M$ any $R$-module, then we denote by $\mathcal{A}(M, B)$ the set of all elements $m$ in $M$ such that $B^{n} m=0$ for some positive integer $n$. Note that $\mathcal{A}(M, B)$ is a submodule of $M$ for every ideal $B$ of $R$. 
Theorem 3.11. Let $R$ be a Dedekind domain which is not a field, and let $F$ denote the field of fractions of $R$. Then an $R$-module $M$ is a $\beta$-module if and only if either

(a) $M$ embeds in the R-module $F$, or

(b) $M \cong E(U)$, the injective envelope of a simple $R$-module $U$, or

(c) $M \cong\left(R / P_{1}^{k_{1}}\right) \oplus \cdots \oplus\left(R / P_{n}^{k_{n}}\right)$ for some positive integers $n, k_{i}$ $(1 \leq i \leq n)$ and distinct maximal ideals $P_{i}(1 \leq i \leq n)$ of $R$.

Proof. Suppose first that $M$ is a $\beta$-module. If $M$ is not torsion, then $M$ is a torsion-free uniform $R$-module (Proposition 3.10), and hence, $M$ embeds in ${ }_{R} F$. Suppose that $M$ is a torsion module. Then $M$ has essential socle, and hence $\operatorname{ann}_{R}(P) \neq 0$ for some maximal ideal $P$ of $R$. Let $P_{i}(i \in I)$ denote the collection of all maximal ideals $P$ of $R$ such that $\operatorname{ann}_{R}(P) \neq 0$. Let $V_{i}=\mathcal{A}\left(M, P_{i}\right)(i \in I)$. Note that, in this case, $M=\oplus_{i \in I} V_{i}$. By Corollary 3.4, the submodule $V_{i}$ has simple socle $U_{i}$ (say) for each $i \in I$. Suppose that $\operatorname{ann}_{R}\left(V_{j}\right)=0$ for some $j \in I$. By Lemma $3.9, V_{j}$ is an essential submodule of $M$, and hence, $M=V_{j}$ and $M$ embeds in $\mathrm{E}\left(U_{j}\right)$. But $\operatorname{ann}_{R}(M)=0$ now gives that $M \cong \mathrm{E}\left(U_{j}\right)$.

This leaves the case that $\operatorname{ann}_{R}\left(V_{i}\right) \neq 0$ for all $i \in I$. Suppose that the index set $I$ is infinite. Let $j \in I$. Then the set $I \backslash\{j\}$ is infinite. Let $W=\oplus_{i \in I \backslash\{j\}} V_{i}$, let $S$ denote the socle of $W$, and let $A=\operatorname{ann}_{R}(S)$. Then $A=\cap_{i \in I \backslash\{j\}} P_{i}$. If $A \neq 0$, then the ring $R / A$ is Artinian, and hence, $R / A$ has only a finite number of maximal ideals. But $P_{i} / A$ is a maximal ideal of the ring $R / A$ for each $i \in I \backslash\{j\}$, a contradiction. Thus, $A=0$, and hence, $V_{j}=0$ by Lemma 3.9 , a contradiction. It follows that the set $I$ is finite. Without loss of generality, we can suppose that $I=\{1,2, \ldots, n\}$ for some positive integer $n$.

Let $1 \leq i \leq n$. Note that $V_{i}$ embeds in $\mathrm{E}\left(U_{i}\right)$. But $\operatorname{ann}_{R}\left(V_{i}\right) \neq 0$ gives that $V_{i} \cong R / P_{i}^{k_{i}}$ for some positive integer $k_{i}$. Thus, $M \cong$ $\left(R / P_{1}^{k_{1}}\right) \oplus \cdots \oplus\left(R / P_{n}^{k_{n}}\right)$, as required.

Conversely, suppose that $M$ satisfies (a), (b) or (c). If (a) holds, then $M$ is a $\beta$-module by Proposition 3.10. If (b) holds, then it is well known that $M$ is a chain module, and hence, $M$ is a $\beta$-module by Corollary 3.6. If (c) holds, then the $R$-module $R / P_{i}^{k_{i}}$ is a chain module for each $1 \leq i \leq n$ and hence $M$ is a $\beta$-module by Corollary 3.6 and Theorem 3.8. Thus, in any case $M$ is a $\beta$-module. 
Corollary 3.12. Let $R$ be a Dedekind domain, and let $M$ be an $R$ module such that $M$ is not torsion-free and the mapping $\beta: \mathcal{L}\left({ }_{R} M\right) \rightarrow$ $\mathcal{L}(R)$ is a homomorphism. Then, the mapping $\beta$ is a monomorphism.

Proof. By Theorem 3.11 and [1, Theorem 3.9], the above mapping $\alpha$ is a surjection, and by Lemma 1.3, the mapping $\beta$ is an injection.

Now we return to consider rings with the property that every module is an $\alpha$-module. Recall that, if a ring $R$ is a domain, then an $R$-module $M$ is called divisible in the case where $M=c M=\{c m: m \in M\}$ for every non-zero element $c$ of $R$. Every injective $R$-module is divisible (see, for example, [16, Proposition 2.6]) and clearly every homomorphic image of a divisible module is divisible. We look again at trivial extensions. Note the following result.

Lemma 3.13. Let $S$ be a domain, let $X$ be a divisible $S$-module, and let $R$ be the trivial extension $X \ltimes S$. Let $B$ be any ideal of $R$. Then $B=X \ltimes I$ for some ideal $I$ of $S$ or $B=Y \ltimes 0$ for some submodule $Y$ of $X$.

Proof. Suppose that $B \subseteq X \ltimes 0$. Then, it is easy to see that $B=Y \ltimes 0$ for some submodule $Y$ of $X$. Now suppose that $B \nsubseteq X \ltimes 0$. Then $B$ contains an element $(s, x)$ for some $0 \neq s \in S, x \in X$. Next $X \ltimes 0=(X \ltimes 0)(s, x) \subseteq B$. It is easy to prove that, in this case, there exists an ideal $I$ of $S$ such that $B=X \ltimes I$.

Theorem 3.14. Let $S$ be a domain, let $X$ be a divisible $S$-module, and let $R=X \ltimes S$ be the trivial extension of $X$ by $S$. Then every $R$-module is an $\alpha$-module if and only if:

(a) every $S$-module is an $\alpha$-module, and

(b) every homomorphic image of $X$ is a $\beta$-module.

Proof. Suppose first that $R$ has the property that every $R$-module is an $\alpha$-module. Let $I$ and $J$ be any ideals of $S$. Let $B=X \ltimes I$ and $C=X \ltimes J$. Then $B$ and $C$ are ideals of $R$. By Corollary 2.13, $R=$ $\left(B:_{R} C\right)+\left(C:_{R} B\right)$. It is easy to check that $\left.B:_{R} C\right)=X \ltimes\left(I:_{S} J\right)$ and $\left(C:_{R} B\right)=X \ltimes\left(J:_{S} I\right)$. It follows that $S=\left(I:_{S} J\right)+\left(J:_{S} I\right)$. 
We have proved that $S=\left(I:_{S} J\right)+\left(J:_{S} I\right)$ for all ideals $I, J$ of $S$. By Corollary 2.13, every $S$-module is an $\alpha$-module. This proves (a).

Now let $Y$ and $Z$ be any submodules of the $S$-module $X$. Let $G=Y \ltimes 0$ and $H=Z \ltimes 0$. Then $G$ and $H$ are ideals of $R$. Note that $\left(G:_{R} H\right)=X \ltimes\left(Y:_{S} Z\right)$ and $\left(H:_{R} G\right)=X \ltimes\left(Z:_{S} Y\right)$. By Corollary 2.13, $R=\left(G:_{R} H\right)+\left(H:_{R} G\right)=X \ltimes\left[\left(Y:_{S} Z\right)+\left(Z:_{S} Y\right)\right]$, and hence, $S=\left(\begin{array}{lll}Y: S & Z\end{array}\right)+\left(\begin{array}{lll}Z: S & Y\end{array}\right)$. We have proved that $S=\left(Y:_{S} Z\right)+\left(Z:_{S} Y\right)$ for all submodules $Y$ and $Z$ of $X$. By Theorem 3.5, every homomorphic image of the $S$-module $X$ is a $\beta$ module. This proves (b).

Conversely, suppose that (a) and (b) hold. Let $E$ and $F$ be any ideals of $R$. If $E \subseteq F$ or $F \subseteq E$, then $R=\left(E:_{R} F\right)+\left(F:_{R} E\right)$. Now suppose that $E \nsubseteq F$ and $F \nsubseteq E$. By Lemma 3.13, either $E+F \subseteq X \ltimes 0$ or $X \ltimes 0 \subseteq E \cap F$. In the first case, $E=U \ltimes 0$ and $F=V \ltimes 0$ for some submodules $U, V$ of the $S$-module $X$. Then

$$
\begin{aligned}
\left(E:_{R} F\right)+\left(F:_{R} E\right) & =\left[X \ltimes\left(U:_{S} V\right)\right]+\left[X \ltimes\left(V:_{S} U\right)\right] \\
& =X \ltimes S=R,
\end{aligned}
$$

because $S=\left(U:_{S} V\right)+\left(V:_{S} U\right)$ by (b) and Theorem 3.5. Now suppose that $X \ltimes 0 \subseteq E \cap F$. Then $E=X \ltimes E^{\prime}$ and $F=X \ltimes F^{\prime}$ for some ideals $E^{\prime}, F^{\prime}$ of $S$. In this case,

$$
\begin{aligned}
\left(E:_{R} F\right)+\left(F:_{R} E\right) & =\left[X \ltimes\left(E^{\prime}:_{S} F^{\prime}\right)\right]+\left[X \ltimes\left(F^{\prime}:_{S} E^{\prime}\right)\right] \\
& =X \ltimes S=R,
\end{aligned}
$$

because $S=\left(E^{\prime}:_{S} F^{\prime}\right)+\left(F^{\prime}:_{S} E^{\prime}\right)$ by (a) and Corollary 2.13. In any case, $R=\left(E:_{R} F\right)+\left(F:_{R} E\right)$, and this holds for all ideals $E$ and $F$ of $R$. By Corollary 2.13, every $R$-module is an $\alpha$-module.

Note that, if $S$ is a Dedekind domain, then it is well known that the injective envelope of any simple $S$-module is a chain module.

Example 3.15. Let $S$ be any Dedekind domain, let $U$ be a simple $S$-module, and let $X$ denote the injective envelope of $U$. Let $R$ denote the ring $X \ltimes S$. Then every $R$-module is an $\alpha$-module.

Proof. By Corollary 2.14, every $S$-module is an $\alpha$-module. The $S$ module $X$ is a chain module, and hence, every homomorphic image of $X$ is a $\beta$-module (Corollary 3.6). Finally, the $S$-module $X$ is a 
divisible module, and hence, by Theorem 3.14, every $R$-module is an $\alpha$-module.

Finally, in this section, we briefly consider semisimple modules.

Proposition 3.16. The following statements are equivalent for a semisimple module $M$ over a ring $R$.

(i) $M$ is a $\beta$-module.

(ii) $M$ is a $\mu$-module.

(iii) $R=\operatorname{ann}_{R}(L)+\operatorname{ann}_{R}(N)$ for all submodules $L$ and $N$ of $M$ with $L \cap N=0$.

Proof. (i) $\Rightarrow$ (iii). By Lemma 3.1.

(iii) $\Rightarrow$ (i). Suppose that (iii) holds. There exist an index set $I$ and simple submodules $U_{i}(i \in I)$ of $M$ such that $M=\oplus_{i \in I} U_{i}$. Let $P_{i}=\operatorname{ann}_{R}\left(U_{i}\right)$ for each $i \in I$. Now let $K$ be any non-zero submodule of $M$. By the argument used in the sufficiency part of the proof of Theorem 3.8 we obtain that

$$
K=\oplus_{i \in I}\left(K \cap U_{i}\right)=\oplus_{i \in I^{\prime}} U_{i}
$$

for some non-empty subset $I^{\prime}$ of $I$.

Now let $L$ and $N$ be any non-zero submodules of $M$. There exists a non-empty subset $I_{1}$ of $I$ such that $L=\oplus_{i \in I_{1}} U_{i}$, and there exists a non-empty subset $I_{2}$ of $I$ such that $N=\oplus_{i \in I_{2}} U_{i}$. Clearly, $L \cap N=$ $\oplus_{i \in I_{1} \cap I_{2}} U_{i}$, and hence, if $B=\operatorname{ann}_{R}(L \cap N)$, then $B=\cap_{i \in I_{1} \cap I_{2}} P_{i}$. It is also clear that

$$
\operatorname{ann}_{R}(L)=\cap_{i \in I_{1}} P_{i}=B \cap C,
$$

where $C=\cap_{i \in I_{1} \backslash I_{2}} P_{i}=\operatorname{ann}_{R}\left(\oplus_{i \in I_{1} \backslash I_{2}} U_{i}\right)$. Similarly, $\operatorname{ann}_{R}(N)=$ $B \cap D$ where $D=\operatorname{ann}_{R}\left(\oplus_{i \in I_{2} \backslash I_{1}} U_{i}\right)$. By hypothesis $R=C+D$, and hence,

$$
\begin{aligned}
\operatorname{ann}_{R}(L \cap N) & =B=B(C+D)=B C+B D \subseteq(B \cap C)+(B \cap D) \\
& =\operatorname{ann}_{R}(L)+\operatorname{ann}_{R}(N) .
\end{aligned}
$$

By Lemma $3.1, M$ is a $\beta$-module.

(ii) $\Leftrightarrow$ (iii). By [17, Proposition 3.16]. 
An example of a semisimple $\beta$-module is given in [17, Example 3.17]. On the other hand, if $I$ is an infinite collection of primes in $\mathbb{Z}$, the ring of rational integers, then the $\mathbb{Z}$-module $\oplus_{p \in I}(\mathbb{Z} / \mathbb{Z} p)$ is not a $\beta$-module by Proposition 3.16. Note further that, if $R$ is a domain, then, for every non-zero ideal $B$ of $R$, the $R$-module $B$ is a $\beta$-module by Lemma 3.1, but if $R$ is not Prüfer there exist finitely generated ideals of $R$ which are not $\mu$-modules over $R$ by [17, Theorem 3.13]. On the other hand, for any ring $R$, the $R$-module $R$ is a $\mu$-module, but if $S$ is a ring and $M$ any non-zero $S$-module, then the ring $R=(M \oplus M) \ltimes S$ has the property that the $R$-module $R$ is not a $\beta$-module by Corollary 3.4. Thus, although the classes of $\beta$-modules and $\mu$-modules coincide for semisimple modules, in general, there is no relationship between them.

4. Anti-monomorphisms and anti-epimorphisms. Let $R$ be a ring and $M$ an $R$-module. The mapping $\alpha: \mathcal{L}(R) \rightarrow \mathcal{L}\left({ }_{R} M\right)$ defined by $\alpha(B)=\operatorname{ann}_{M}(B)$, for every ideal $B$ of $R$, can be an antihomomorphism (that is, $M$ is an $\alpha$-module) without it being either an anti-monomorphism or an anti-epimorphism. For example, let $R$ be a domain which is not a field, and let $M$ be a non-zero torsion-free $R$-module. By Corollary 2.11, $M$ is an $\alpha$-module. For any non-zero ideal $B$ of $R, \alpha(B)=\operatorname{ann}_{M}(B)=0$ and $\alpha(0)=\operatorname{ann}_{M}(0)=M$. Let $a$ be a non-zero element of $R$ which is not a unit. Then $R a \neq R a^{2}$, but $\alpha(R a)=\alpha\left(R a^{2}\right)$. Thus, $\alpha$ is not an injection. Note next that $M$ is not a simple $R$-module. Let $K$ be any proper non-zero submodule of $M$. Then $K \neq \alpha(C)$ for any ideal $C$ of $R$. Thus, $\alpha$ is not a surjection.

Next let $R$ be any ring which is not a field, and let $U$ be any simple $R$-module with $P=\operatorname{ann}_{R}(U)$. In this case, for any ideal $B$ of $R$, $\alpha(B)=\operatorname{ann}_{U}(B)=U$ if $B \subseteq P$ and $\alpha(B)=0$ otherwise. We have already seen that $U$ is an $\alpha$-module (Corollary 2.9). However, $\alpha(0)=\alpha(P)=U$ shows that $\alpha$ is not an injection. But $\alpha$ is clearly a surjection. Thus, $\alpha$ is an anti-epimorphism but not an antimonomorphism. Later, we shall give an example to show that the mapping $\alpha$ can be an anti-monomorphism but not an anti-epimorphism.

Recall that a module $M$ is a comultiplication module for a ring $R$ when the mapping $\alpha: \mathcal{L}(R) \rightarrow \mathcal{L}\left({ }_{R} M\right)$ is a surjection (but not necessarily an anti-epimorphism). We next consider when the direct sum of modules is a comultiplication module. First, we prove two lemmas: 
Lemma 4.1. Let $R$ be a ring, and let an $R$-module $M=M_{1} \oplus M_{2}$ be a direct sum of submodules $M_{1}, M_{2}$. Suppose that $M$ is a comultiplication module. Then $\operatorname{ann}_{M_{2}}\left(\operatorname{ann}_{R}\left(M_{1}\right)\right)=0$.

Proof. Let $B=\operatorname{ann}_{R}\left(M_{1}\right)$. By [1, Theorem 1.5], we have:

$$
M_{1}=\operatorname{ann}_{M}(B)=\operatorname{ann}_{M_{1}}(B) \oplus \operatorname{ann}_{M_{2}}(B),
$$

and hence $\operatorname{ann}_{M_{2}}(B)=0$, as required.

Lemma 4.2. Let $R$ be a ring, and let an $R$-module $M=M_{1} \oplus M_{2}$ be a direct sum of submodules $M_{1}, M_{2}$ such that $\operatorname{ann}_{M_{1}}\left(\operatorname{ann}_{R}\left(M_{2}\right)\right)=0$ and $M_{1}$ is a comultiplication module. Let a submodule $N=N_{1} \oplus N_{2}$ of $M$ be a direct sum of submodules $N_{1}$ of $M_{1}$ and $N_{2}$ of $M_{2}$. Let $B_{i}=\operatorname{ann}_{R}\left(N_{i}\right)(i=1,2)$. Then $\pi_{1}\left(\operatorname{ann}_{M}\left(B_{1} \cap B_{2}\right)\right) \subseteq N_{1}$, where $\pi_{1}: M \rightarrow M_{1}$ is the canonical projection.

Proof. Suppose that $m \in M$ satisfies $\left(B_{1} \cap B_{2}\right) m=0$. There exist elements $m_{i} \in M_{i}(i=1,2)$ such that $m=m_{1}+m_{2}$. Clearly $\left(B_{1} \cap B_{2}\right) m_{1}=0$ and, in this case, $B_{2}\left(B_{1} m_{1}\right)=0$. But $N_{2} \subseteq M_{2}$ implies that $\operatorname{ann}_{R}\left(M_{2}\right) \subseteq \operatorname{ann}_{R}\left(N_{2}\right)=B_{2}$, which in turn implies that

$$
B_{1} m_{1} \subseteq \operatorname{ann}_{M_{1}}\left(B_{2}\right) \subseteq \operatorname{ann}_{M_{1}}\left(\operatorname{ann}_{R}\left(M_{2}\right)\right)=0 .
$$

Thus, $m_{1} \in \operatorname{ann}_{M_{1}}\left(B_{1}\right)=N_{1}$ by [1, Theorem 1.5].

The following result generalizes [1, Theorem 3.1].

Theorem 4.3. Let $R$ be a ring, and let the $R$-module $M=\oplus_{i \in I} M_{i}$ be a direct sum of submodules $M_{i}(i \in I)$. Then the $R$-module $M$ is a comultiplication module if and only if

(a) the R-module $M_{j}$ is a comultiplication module for all $j \in I$,

(b) $N=\oplus_{i \in I}\left(N \cap M_{i}\right)$ for every submodule $N$ of $M$, and

(c) $\operatorname{ann}_{M_{j}}\left(\operatorname{ann}_{R}\left(\oplus_{i \neq j} M_{i}\right)\right)=0$ for all $j \in I$.

Proof. Suppose first that the $R$-module $M$ is a comultiplication module. Then (a) and (b) follow by [1, Lemma 2.1] and (c) by Lemma 4.1.

Conversely, suppose that (a), (b) and (c) hold. Let $N$ be any submodule of $M$, and let $N_{i}=N \cap M_{i}(i \in I)$. By (b), $N=$ 
$\oplus_{i \in I} N_{i}$. Let $B_{i}=\operatorname{ann}_{R}\left(N_{i}\right)(i \in I)$, and let $B=\cap_{i \in I} B_{i}$. Then $B N=\oplus_{i \in I} B N_{i}=0$, so that $N \subseteq \operatorname{ann}_{M}(B)$. Let $m \in \operatorname{ann}_{M}(B)$. Let $j \in I$, and let $\pi_{j}: M \rightarrow M_{j}$ denote the canonical projection. If $C=\cap_{i \neq j} B_{i}$, then $B=B_{j} \cap C$ and $C=\operatorname{ann}_{R}\left(\oplus_{i \neq j} N_{i}\right)$. By (a), $M_{j}$ is a comultiplication module and, by (c), $\operatorname{ann}_{M_{j}}\left(\oplus_{i \neq j} M_{i}\right)=0$. Therefore, we can apply Lemma 4.2 to obtain $\pi_{j}(m) \in N_{j}$. It follows that $m \in \oplus_{i \in I} N_{i}=N$. Hence, $\operatorname{ann}_{M}(B) \subseteq N$, and we have proved that $N=\operatorname{ann}_{M}(B)$. It follows that the $R$-module $M$ is a comultiplication module.

Corollary 4.4. Let $R$ be a ring, and let an $R$-module $M=\oplus_{i \in I} M_{i}$ be a direct sum of submodules $M_{i}(i \in I)$ such that $R=\operatorname{ann}_{R}\left(M_{j}\right)+$ $\operatorname{ann}_{R}\left(\oplus_{i \neq j} M_{i}\right)$ for all $j \in I$. Then the $R$-module $M$ is a comultiplication module if and only if the $R$-module $M_{i}$ is a comultiplication module for all $i \in I$.

Proof. It is not difficult to show that the module $M$ satisfies (b) and (c) in Theorem 4.3, and, therefore, the result follows immediately from Theorem 4.3.

Given a ring $R$, an $R$-module $M$ is a comultiplication $\alpha$-module if and only if the mapping $\alpha: \mathcal{L}(R) \rightarrow \mathcal{L}\left({ }_{R} M\right)$ is an anti-epimorphism. Combining Proposition 2.6 and Theorem 4.3 we have the next result without further proof.

Corollary 4.5. Let $R$ be a ring, and let the $R$-module $M=\oplus_{i \in I} M_{i}$ be a direct sum of submodules $M_{i}(i \in I)$. Then the $R$-module $M$ is a comultiplication $\alpha$-module if and only if:

(a) the $R$-module $M_{j}$ is a comultiplication $\alpha$-module for all $j \in I$,

(b) $N=\oplus_{i \in I}\left(N \cap M_{i}\right)$ for every submodule $N$ of $M$, and

(c) $\operatorname{ann}_{M_{j}}\left(\operatorname{ann}_{R}\left(\oplus_{i \neq j} M_{i}\right)\right)=0$ for all $j \in I$.

Proposition 4.6. Let $R$ be a ring, and let an $R$-module $M=\oplus_{i \in I} U_{i}$ be a direct sum of simple submodules $U_{i}(i \in I)$ for some non-empty index set $I$. Let $P_{i}=\operatorname{ann}_{R}\left(U_{i}\right)(i \in I)$. Then $M$ is a comultiplication $\alpha$-module if and only if $R=P_{j}+\left(\cap_{i \neq j} P_{i}\right)$ for all $j \in I$.

Proof. By Corollaries 2.9 and 4.4 . 
Now we look at a situation when the mapping $\alpha$ is an antimonomorphism. Let $R$ be any ring, and let $B$ be any ideal of $R$. Then $\mathcal{V}(B)$ will denote the collection of all prime ideals $P$ of $R$ with $B \subseteq P$. Note that $\mathcal{V}(R)$ is the empty set and $\mathcal{V}(0)$ is the collection of all prime ideals of $R$. This brings us to the following result.

Theorem 4.7. Let $R$ be a ring, and let $M$ denote the $R$-module $\oplus_{P \in \mathcal{V}(0)}(R / P)$. Then the following statements are equivalent:

(i) $R$ is von Neumann regular.

(ii) The mapping $\alpha: \mathcal{L}(R) \rightarrow \mathcal{L}\left({ }_{R} M\right)$ is an anti-monomorphism.

(iii) The mapping $\alpha: \mathcal{L}(R) \rightarrow \mathcal{L}\left({ }_{R} M\right)$ is an injection.

Proof. (i) $\Rightarrow$ (ii). Because $R$ is a von Neumann regular ring, every prime ideal of $R$ is maximal. Next note that $M$ is an $\alpha$-module by Corollary 2.9. Let $B$ be any ideal of $R$. Then $\alpha(B)=\operatorname{ann}_{M}(B)=$ $\oplus_{P \in \mathcal{V}(B)}(R / P)$. If $C$ is any ideal of $R$ with $\alpha(C)=\alpha(B)$, then $\mathcal{V}(B)=\mathcal{V}(C)$. But this implies

$$
B=\cap_{P \in \mathcal{V}(B)} P=\cap_{P \in \mathcal{V}(C)} P=C,
$$

by [2, Example 18, number 23]. Thus, $\alpha(B)=\alpha(C)$ implies that $B=C$. It follows that $\alpha$ is an anti-monomorphism.

(ii) $\Rightarrow$ (iii). Clear.

(iii) $\Rightarrow$ (i). Suppose that the mapping $\alpha: \mathcal{L}(R) \rightarrow \mathcal{L}\left({ }_{R} M\right)$ is an injection. Let $a \in R$. Then $\mathcal{V}(R a)=\mathcal{V}\left(R a^{2}\right)$, and hence,

$$
\alpha(R a)=\oplus_{P \in \mathcal{V}(R a)}(R / P)=\oplus_{P \in \mathcal{V}\left(R a^{2}\right)}(R / P)=\alpha\left(R a^{2}\right) .
$$

Because $\alpha$ is an injection, the ideal $R a=R a^{2}$, and hence, $a=a b a$ for some $b \in R$. Thus, $R$ is a von Neumann regular ring.

This allows us to produce examples to show that the mapping $\alpha$ can be an anti-monomorphism without being an anti-epimorphism.

Corollary 4.8. Let $R$ be a von Neumann regular ring, let $P_{i}(i \in$ $I)$ denote the collection of all maximal ideals of $R$, and let $M=$ $\oplus_{i \in I}\left(R / P_{i}\right)$. Then the mapping $\alpha: \mathcal{L}(R) \rightarrow \mathcal{L}\left({ }_{R} M\right)$ is an antimonomorphism. Moreover, $\alpha$ is an anti-epimorphism (and hence an anti-isomorphism) if and only if $R$ is semiprime Artinian. 
Proof. By Theorem 4.7, $\alpha$ is an anti-monomorphism. Now suppose that the $R$-module $M$ is a comultiplication module. Let $j \in I$. By Proposition 4.6, $R=P_{j}+\left(\cap_{i \neq j} P_{i}\right)$. But $\cap_{i \in I} P_{i}=0$. Thus, $P_{j}=R e_{j}$ for some idempotent element $e_{j}$ of $R$. We have proved that every maximal ideal of $R$ is generated by an idempotent. Thus, $R$ has no proper essential ideals and hence is semiprime Artinian. Conversely, if $R$ is semiprime Artinian, then $\alpha$ is a surjection by Proposition 4.6.

\section{REFERENCES}

1. Y. Al-Shaniafi and P.F. Smith, Comultiplication modules over commutative rings, J. Comm. Alg. 3 (2011), 1-29.

2. F.W. Anderson and K.R. Fuller, Rings and categories of modules, SpringerVerlag, New York, 1974.

3. H. Ansari-Toroghy and H. Farshadifar, The dual notion of multiplication modules, Taiwan. J. Math. 11 (2007), 1189-1201.

4. Comultiplation modules and related results, Honam Math. J. 30 (2008), 91-99.

5. - On comultiplication modules, Kor. Ann. Math. 25 (2008), 57-66.

6. J. Dauns, Prime modules, J. reine angew Math. 298 (1978), 156-181.

7. R. Gilmer, Multiplicative ideal theory, Marcel Dekker, New York, 1972.

8. C.R. Hajarnavis and N.C. Norton, On dual rings and their modules, J. Alg. 93 (1985), 253-266.

9. A. Harmanci, P.F. Smith and Y. Tiras, A characterization of prime submodules, J. Alg. 212 (1999), 743-752.

10. I. Kaplansky, Dual rings, Ann. Math. 49 (1948), 689-701.

11. C.-P. Lu, Prime submodules of modules, Comm. Math. Univ. Sanct. Paul. 33 (1984), 61-69.

12. R.L. McCasland and M.E. Moore, Prime submodules, Comm. Alg. 20 (1992), 1803-1817.

13. R.L. McCasland and P.F. Smith, Prime submodules of Noetherian modules, Rocky Mountain J. Math. 23 (1993), 1041-1062.

14. W.K. Nicholson and M.F. Yousif, On dual rings, New Zealand J. Math. 28 (1999), 65-70.

15. Y. Shaniafi and P.F. Smith, Comultiplication modules over commutative rings II, J. Comm. Alg. 4 (2012), 153-174.

16. D.W. Sharpe and P. Vamos Injective modules, Cambr. Tracts Math. Math. Phys. 62, Cambridge University Press, Cambridge, 1972.

17. P.F. Smith, Mappings between module lattices, Int. Electr. J. Alg. 15 (2014), $173-195$.

18. Complete homomorphisms between module lattices, Int. Electr. J. Alg. 16 (2014), 16-31. 
Department of Mathematics, University of Glasgow, Glasgow, G12 8QW, SCOTLAND UK

Email address: patrick.smith@glasgow.ac.uk 